\title{
Efecto del entorno y rendimiento académico en las es trategias de afrontamiento
}

\section{infantil}

\section{Environmental effect and academic performance in child confrontation strategies}

\author{
Francisco Manuel Morales Rodríguez*, Tamara García Medina** \\ *Universidad de Granada **Universidad de Málaga
}

\begin{abstract}
Resumen
Los objetivos de esta investigación son analizar diferencias en las estrategias de afrontamiento del estrés cotidiano empleadas por escolares en función de la procedencia de escuela urbana vs rural y rendimiento académico. Participaron 126 escolares entre 9 y 12 años de la provincia de Málaga. Se aplicó una Escala de Afrontamiento para Niños para evaluar estrategias de afrontamiento. El diseño de investigación utilizado en función de los objetivos es de carácter cuantitativo. Los resultados encuentran diferencias estadísticamente significativas en el tipo de estrategias de afrontamiento en función de la procedencia y rendimiento académico.

Palabras clave: Afrontamiento, estrés cotidiano, niños, procedencia de escuela urbana vs. rural, rendimiento académico
\end{abstract}

\begin{abstract}
The present research aims to analyse differences in strategies of coping with daily stress according to urban vs rural area and academic performance. Participants are 126 boys and girls aged 9 to 12 years-old from the province of Malaga, who were administered the Coping Scale for Children (EAN in Spanish). The research design used according to the expressed objectives is of quantitative nature. Results show statistically significant differences in the type of coping strategies used depending on the living environment and academic performance. Keywords: Academic performance, Children, Coping, Everyday Stressors, urban vs rural área
\end{abstract}

Desde el Modelo de Afrontamiento Transaccional sobre el estrés que se defiende en este trabajo, se considera que el estrés implica tanto a la persona como al medio en el que está inmerso, y se asume la siguiente definición: "Estrés psicológico implica una particular relación entre la persona y el medio que es evaluado como agotador o que excede sus recursos y pone en peligro su bienestar" (Lazarus y Folkman, 1986, p.43). Otros enfoques (García-Martín y Jiménez-Hernández, 1995, p.86), en esta misma línea, integran 3 perspectivas desde las que abordar este constructo: “a) estrés como estímulo o acontecimiento estimular, b) estrés como respuesta adoptada por el organismo y c) estrés como interacción entre organismo y su entorno".

Desde el modelo transaccional anteriormente señalado el afrontamiento se define como "aquellos esfuerzos cognitivos y conductuales constantemente cambiantes que se desarrollan para manejar las demandas específicas externas y/o internas que son evaluadas como excedentes o desbordantes de los recursos del individuo" (Lazarus y Folkman, 1986, p. 164).

Otros estudios, coherentes con el enfoque situacional defendido en el presente trabajo, como el de Cohen y Lazarus (1979), asumen una perspectiva transaccional y definen el afrontamiento como una serie de esfuerzos con el fin solventar aquellas demandas propias y del ambiente que la persona ve como una situación problemática. Las estrategias de afrontamiento son consideradas como las acciones conductuales o cognitivas en las que se implica una persona (Compas, 1987; Morales y Trianes, 2012).Una clasificación reciente de las estrategias de afrontamiento es la que realizan autores como Espada y Grau (2012) que distinguen entre estrategias de afrontamiento activas/productivas (búsqueda de información, de distracciones o aplicar pensamiento positivo) y estrategias pasivas e improductivas tales como tratar de aislarse o, directamente, evitar el problema.

En otra investigación sobre afrontamiento del estrés cotidiano infantil (Morales-Rodríguez et al. 2012) se incluye estrategias de afrontamiento productivo ("Solución activa", "Contar el problema a otros", "Búsqueda de información y guía" y "Actitud positiva") e improductivo ("Indiferencia", "Conducta agresiva", "Reservarse el problema para sí", "Evitación cognitiva" y "Evitación conductual") ante problemas relacionados con el contexto familiar, salud, las tareas escolares y las relaciones sociales.

En este sentido, clasificaciones relevantes y consolidadas son las que se orientan hacia dos tipos de afrontamiento en función de las consecuencias positivas o negativas para las personas. Hay autores que defienden que el empleo del afrontamiento dirigido a solucionar el problema es saludable mientras que otras estrategias improductivas y evitativas serían contraproducentes para las personas (Seiffge-Krenke, 2000).

Dado que existen factores como el contexto de procedencia, el sexo, la edad y el rendimiento académico que pueden influir en las estrategias de afrontamiento empleadas por niños y niñas; los mismos se han seleccionado en esta investigación como el conjunto de variables relevantes que pueden producir diferencias en las estrategias de afrontamiento utilizadas que pueden 
relacionarse con diferentes resultados de adaptación y salud mental en niños y adolescentes (Morales y Trianes, 2012).

Respecto a la procedencia de la muestra, la investigación realizada por Hernández-Pereyra (2004) demuestra que ante una situación estresante aquellas personas que proceden del ámbito urbano suelen utilizar en mayor medida estrategias de tipo activo y productivas de afrontamiento, en comparación con las que proceden del ámbito rural. Sin embargo, existen datos contradictorios como trabajos que señalan que quizás en aldeas y pueblos se puede producir mayor calidad en las relaciones interpersonales que puede relacionarse con estrategias de afrontamiento más eficaces y mayor bienestar infantil (Rostosky y Riggle, 2015) y otros estudios que no encuentran un papel claro del entorno en la generación de diferentes estrategias (Elgar, Arlett y Groves, 2003).

Respecto a las diferencias en el afrontamiento en función del rendimiento académico o calificaciones obtenidas, los resultados no son consistentes. Algún estudio como el de Castro-Solano (2005) encuentra que los grupos de rendimiento medio y alto utilizaban, con mayor frecuencia, estrategias dirigidas a resolver el problema. En cambio, otro trabajo (Rovella y Sans, 2003) no encuentra diferencias estadísticamente significativas en las estrategias de afrontamiento empleadas en función de las calificaciones obtenidas.

En el presente trabajo se evalúa el rendimiento en la asignatura de Matemáticas ya que el educando al enfrentarse al aprendizaje de los conceptos matemáticos y el rendimiento académico demandado suele tener una serie de dificultades que pueden generar estrés y percepción de fracaso en estudiantes de Educación Primaria (Trianes, 1999). Otros estudios (Sánchez, Segovia y Miñán, 2011) en esta línea señalan que aunque el aprendizaje de las matemáticas en Educación Primaria es una tarea fundamental en distintos ámbitos de la vida, se trata de una de las asignaturas en las que se produce mayor fracaso escolar y estrés académico; encontrándose frecuentemente en el alumnado actitudes negativas, aversión y rechazo hacia dicha materia. En otras investigaciones (Massone y González, 2003) también se pone de manifiesto que las dificultades hacia las matemáticas representan un problema relevante en el ámbito educativo y de ahí la necesidad de identificar los factores y estrategias de afrontamiento empleadas por los escolares en esta situación. Precisamente en nuestro estudio (contexto rural) se corroboró desde el inicio que es la asignatura donde existe menor rendimiento, y genera mayores dificultades según informan padres y maestros; lo que puede generar mayor estrés y donde se requiere evaluar las estrategias utilizadas, generar toma de conciencia sobre las mismas y potenciar estrategias productivas de afrontamiento.

Existen investigaciones consolidadas como, por ejemplo, las de Frydenberg (1997) que se han centrado en otras variables como el afrontamiento del estrés en función del sexo encontrando que las chicas muestran más preocupación, suelen hacer uso de una mayor variedad de estrategias de afrontamiento y emplean más la estrategia búsqueda de apoyo social mientras que los chicos suelen utilizar más estrategias de tipo evitativo como buscar distracción. También existen estudios que encuentran que los niños seleccionan estrategias de estilo evitativo y de apoyo de los amigos mientras que las chicas recurren más a estrategias de apoyo de los familiares (Giménez-Gualdo, Arnaiz-Sánchez y Maquilon-Sánchez, 2013).

En el ámbito educativo del afrontamiento infantil, en nuestro contexto es necesario profundizar en las posibles diferencias en el empleo de estrategias de afrontamiento en función de variables como entorno de procedencia y el rendimiento académico para proceder al diseño de intervenciones psicoeducativas más eficaces.

Los objetivos del presente trabajo son:

a) Analizar diferencias en el empleo de estrategias de afrontamiento del estrés cotidiano empleadas por niños y niñas con una edad comprendida entre 9 y 12 años en función del ámbito de procedencia (urbano o rural);

b) Analizar posibles diferencias en el empleo de estrategias de afrontamiento en función del rendimiento académico en la asignatura de Matemáticas.

\section{Método}

\section{Participantes}

En el estudio participaron un total de 126 niños y niñas de Centros de Educación Primaria de la provincia de Málaga con edades comprendidas entre los 9 y 12 años.

Del total de la muestra 78 niños y niñas (31 niñas y 47 niños) pertenecen a un colegio del núcleo urbano de la zona costera de la provincia de Málaga que cursan $4^{\circ}$ (26), $5^{\circ}(26)$ y $6^{\circ}(26)$ de primaria. También fueron participantes 48 niños y niñas de un colegio de ámbito rural de la provincia de Málaga. En este último grupo, la muestra está formada por 16 niños y 32 niñas. De ellos, 24 son de $5^{\circ}$ de primaria y 24 son de $6^{\circ}$ de primaria. Participaron alumnos matriculados en $\operatorname{los}$ años $4^{\circ}$ a $6^{\circ}$ en el nivel de educación primaria excluido el alumnado con Necesidades Específicas de Apoyo Educativo derivadas de discapacidad o trastornos graves de conducta.

Los centros educativos corresponden a zonas con un nivel socioeconómico medio.

La selección de la muestra se llevó a cabo con un muestreo de tipo incidental. La muestra está equilibrada según el sexo.

\section{Instrumento}

Escala de Afrontamiento para Niños (EAN) (Morales-Rodríguez et al., 2012). Esta herramienta de medición evalúa el afrontamiento del estrés cotidiano en niños y niñas en un rango de edad comprendido entre 8 y 12 años.

La escala cuenta con un total de 35 ítems que se agrupan en cuatro grupos de ítems referidos a diferentes situaciones problemáticas a las que se debe enfrentar un niño en su vida diaria. El primero de estos grupos, está formado por preguntas referidas a los problemas en casa y está compuesto por un total de nueve ítems. El segundo grupo está formado por ocho ítems, en este caso se describen situaciones en las que el niño enferma y debe ir 
al médico. El tercer grupo representa diferentes estrategias que pueden ser utilizadas ante situaciones relacionadas con lo académico y se compone de nueve ítems. El cuarto grupo cuenta con diversas estrategias de afrontamiento para una situación conflictiva que implica problemas con los compañeros de clase.

Cada uno de esos grupos de ítems incluye estrategias de afrontamiento del estrés que pueden categorizarse como Afrontamiento Productivo o Afrontamiento Improductivo.

Por un lado, dentro de las estrategias productivas se encuentran una suma de 16 ítems. El Afrontamiento Productivo incluye aquellas que implican la "Solución activa del problema", "Comunicar el problema a otros", "Búsqueda de información y guía" y "Actitud positiva". Por otro lado, las estrategias improductivas suman un total de 19 ítems y se refieren a actitudes que no contribuyen a una solución eficaz del conflicto planteado; tales como "Indiferencia", "Conducta agresiva", "Reservarse el problema para sí mismo", "Evitación cognitiva" y "Evitación conductual".

Los ítems que componen esta escala son evaluados mediante una escala de respuesta de tipo Likert que incluye 3 opciones de respuesta por orden de frecuencia de dicha estrategia de afrontamiento: Nunca, Algunas veces y Muchas veces.

Esta escala ha demostrado buenas propiedades psicométricas. Estos resultados psicométricos son avalados por la prueba de análisis confirmatorio en la que todos los parámetros son estadísticamente significativos $(p<.05)$. Además se emplearon pruebas como Alpha de Cronbach (cuyos valores oscilan entre .41 y .85 según la estrategia, coeficiente de fiabilidad Rho (valores que oscilan entre .44 y .87 según estrategia) y fiabilidad test-retest (valores entre .47 y .55 según estrategia).

\section{Procedimiento}

En primer lugar, se procedió a informar a los responsables de los escolares de los dos colegios solicitando el permiso y el consentimiento informado de los padres, madres o tutores legales de los niños. Luego, se acudió a los dos centros, de la zona de costa y del interior de la provincia de Málaga, para aplicar la escala EAN.

La escala se aplicó de forma colectiva en las distintas clases de Educación Primaria $\left(4^{\circ}, 5^{\circ}\right.$ y $6^{\circ}$ de primaria) por un único investigador en colaboración con los tutores académicos del alumnado.

En todos los grupos se aplicó la prueba leyendo los ítems en voz alta, para lograr una mejor comprensión por parte del alumnado y resolver posibles dudas.

\section{Análisis de datos}

El diseño de investigación utilizado en función de los objetivos planteados es de carácter cuantitativo ya que incluye aspectos cuantitativos en su metodología utilizando un cuestionario con formato de respuesta tipo Likert. El presente trabajo es un estudio ex post facto según el sistema de clasificación de las metodologías de investigación señalado por Montero y León (2007) ya que no se manipularon las variables ni el contexto real de investigación.
Se realizaron análisis descriptivos (medias, deviaciones típicas y porcentajes) para evaluar las estrategias de afrontamiento empleadas por los escolares.

Se llevaron a cabo varios análisis estadísticos, $t$-Student para muestras independientes y ANOVA, con el programa estadístico SPSS para analizar si existen diferencias estadísticamente significativas en el empleo de estrategias de afrontamiento en función del ámbito de procedencia (urbana o rural). Para analizar diferencias en el empleo de estrategias de afrontamiento en función del rendimiento académico se utilizó el ANOVA no paramétrico de Kruskal-Wallis.

\section{Resultados}

A continuación, en la Tabla 1 se muestran los resultados de aplicar la $t$-Student para muestras independientes con objeto de averiguar la existencia de diferencias estadísticamente significativas entre las medias en el empleo de estrategias de afrontamiento del estrés cotidiano en función del entorno. La variable "entorno" se ha categorizado en dos grupos: urbano $(n=78)$ y rural $(n=48)$.

Se ha obtenido que las estrategias de afrontamiento "Indiferencia", "Evitación conductual", "Evitación cognitiva", "Contar el problema a otros" y "Búsqueda de información y guía", muestran diferencias estadísticamente significativas entre los grupos urbano y rural. En el caso de las estrategias de afrontamiento "Conducta agresiva", "Reservarse el problema para sí", "Solución activa", y "Actitud positiva" no se demuestra la existencia de diferencias estadísticamente significativas entre las medias de ambos grupos.

Concretamente, puede destacarse que los escolares de ámbito rural emplean en mayor medida que los de ámbito urbano la estrategia "Actitud positiva" mientras que las otras estrategias en la que se encuentran diferencias significativas son más utilizadas en la población urbana.

Tabla 1

Diferencia de medias en el empleo de estrategias de afrontamiento en función del entorno

\begin{tabular}{|c|c|c|c|}
\hline \multirow[b]{2}{*}{$\begin{array}{l}\text { Estrategias de } \\
\text { afrontamiento }\end{array}$} & Urbano & Rural & \multirow[b]{2}{*}{$\begin{array}{c}g l=55 \\
\mathrm{t}\end{array}$} \\
\hline & $\begin{array}{l}\text { Media } \\
(d t)\end{array}$ & $\begin{array}{c}\text { Media } \\
(d t)\end{array}$ & \\
\hline Indiferencia & $\begin{array}{l}1.36 \\
(.39)\end{array}$ & $\begin{array}{l}1.11 \\
(.36)\end{array}$ & $3.59 * *$ \\
\hline Conducta agresiva & $\begin{array}{l}1.38 \\
(.42)\end{array}$ & $\begin{array}{l}1.23 \\
.47)\end{array}$ & 1.78 \\
\hline $\begin{array}{l}\text { Reservarse el problema } \\
\text { para sí }\end{array}$ & $\begin{array}{l}1.68 \\
(.50)\end{array}$ & $\begin{array}{l}1.65 \\
(.73)\end{array}$ & .31 \\
\hline Evitación cognitiva & $\begin{array}{l}1.94 \\
(.53)\end{array}$ & $\begin{array}{l}1.60 \\
(.62)\end{array}$ & $3.25 * *$ \\
\hline Evitación conductual & $\begin{array}{l}1.90 \\
(.49)\end{array}$ & $\begin{array}{l}1.56 \\
(.54)\end{array}$ & $3.56^{* *}$ \\
\hline Solución activa & $\begin{array}{l}2.35 \\
(.39)\end{array}$ & $\begin{array}{l}2.34 \\
(.60)\end{array}$ & .13 \\
\hline $\begin{array}{l}\text { Contar el problema a } \\
\text { otros }\end{array}$ & $\begin{array}{l}2.25 \\
(.43)\end{array}$ & $\begin{array}{l}2.00 \\
(.75)\end{array}$ & $2.41 *$ \\
\hline $\begin{array}{l}\text { Búsqueda de información } \\
\text { y guía }\end{array}$ & $\begin{array}{l}2.22 \\
(.51)\end{array}$ & $\begin{array}{l}1.94 \\
(.63)\end{array}$ & $2.69 * *$ \\
\hline Actitud positiva & $\begin{array}{l}2.27 \\
(.48) \\
\end{array}$ & $\begin{array}{l}2.48 \\
(.66)\end{array}$ & $-2.00 *$ \\
\hline
\end{tabular}


A continuación se señalan los resultados del análisis Chi-Cuadrado entre las estrategias de afrontamiento del estrés cotidiano empleadas en función de las calificaciones que han obtenido en Matemáticas en el contexto rural. Es el contexto y asignatura en el que se ha producido un menor rendimiento con evidencias de mayor grado de dificultad y estrés académico según perciben padres y maestros. Solo se presentan los resultados estadísticamente significativos, comparando las calificaciones de Insuficiente y Sobresaliente.

En este caso se han agrupado los ítems en función de las situaciones conflictivas más frecuentes a la que se enfrentan las personas durante la infancia: "Cuando hay algún problema en casa", "Cuando me pongo enfermo/a y tengo que ir al médico", "Cuando tengo problemas con algún compañero de clase" y "Cuando tengo problemas con las tareas escolares".

Se ha categorizado la variable de calificaciones en grupos: Insuficiente $(n=2)$, Suficiente $(n=0)$, Bien $(n=1)$, Notable $(n=4)$ y Sobresaliente $(n=6) ; \quad(N=13))$. Posteriormente, los análisis comparan los problemas que tratan de solventar mediante las estrategias de afrontamiento del estrés cotidiano y la variable calificación de matemáticas mediante ANOVA no paramétrico en el contexto y asignatura en la que se han producido y percibido más dificultades/problemas con las notas. Los datos analizados muestran cómo los ítems correspondientes a los problemas relacionados con "Cuando tengo problemas con algún compañero/a de clase" muestran diferencias estadísticamente significativas en función de las calificaciones obtenidas. Aunque, en el caso de las situaciones: "Cuando hay algún problema en casa", "Cuando me pongo enfermo/a y tengo que ir al médico" y "Cuando tengo problemas con las tareas escolares" no se ha encontrado diferencias significativas en función de las calificaciones.

Se encuentran diferencias estadísticamente significativas en las situaciones en las que aparece un conflicto con los compañeros de clase y, en consecuencia, en las estrategias que los niños y niñas utilizan en esa situación; lo cual guarda relación con la calificación que obtienen. Dentro de las estrategias utilizadas en esta situación, las estrategias productivas ("Solución activa", "Comunicar el problema a otros", "Búsqueda de información y guía" y "Actitud positiva") son menos utilizadas por aquellos que tienen la calificación de Insuficiente que por el resto de niños y niñas que obtienen Sobresaliente.

\section{Discusión}

Los resultados de este estudio evidencian que el entorno de procedencia de la muestra es relevante en la utilización de diferentes estrategias de afrontamiento. Las estrategias de "Indiferencia", "Evitación conductual", "Evitación cognitiva", "Contar el problema a otros" y "Búsqueda de información y guía" son más utilizadas por escolares de ámbito urbano en comparación con los de entorno rural; resultado que parece congruente con otro estudio (Hernández-Pereyra, 2004) en el que la muestra urbana utiliza más estrategias productivas de afrontamiento ("Contar el problema a otros"; "Búsqueda de Información y Guía") en comparación con la rural. Se ha demostrado que la estrategia "Búsqueda de Información y Guía", que implica intentos de aproximación al problema, forma parte de un estilo funcional de afrontamiento (Seiffge-Krenke y Shulman, 1990) mientras que las estrategias no productivas tales como "Indiferencia" y "Evitación" se relacionan con un estilo disfuncional de afrontamiento (Morales y Trianes, 2012).

El presente trabajo muestra que los escolares con menor rendimiento académico en la asignatura de Matemáticas, han utilizado menos estrategias productivas que implican la solución activa del problema. Otros autores muestran también que los escolares con un menor rendimiento académico emplean estrategias de afrontamiento más improductivas (Castro-Solano, 2005).

\section{Referencias}

Castro-Solano, A. (2005). Estilos de personalidad, afrontamiento e inteligencia como predictores de las trayectorias académicas de cadetes de instrucción militar. Anuario de Psicología, 36(2), 197-210. Recuperado

de: http://www.scielo.cl/scielo.php?script=sci_nlinks\&ref $=4102577 \&$ pid $=$ S0718-2228200500010000200013\&1 ng=es

Cohen, F. y Lazarus, R. S. (1979). Coping with the stresses of illness. En G. C. Stone, F. Cohen, N. E. Adler, \& Associates (Eds.), Health Psychology - A Handbook: Theories, Applications, and Challenges of a Psychological Approach to the Health Care System (pp. 217-254). San Francisco: Jossey-Bass.

Compas, B. E. (1987). Coping with stress during childhood and adolescence.Psychological Bulletin, 101 , 393-403. http://dx.doi.org/10.1037/0033-2909.127.1.87

Elgar, F. J., Arlett, C. y Groves, R. (2003).Stress, coping, and behavioural problems among rural and urban adolescents.Journal of Adolescence, 26, 574-585. http://dx.doi.org/10.1016/S0140-1971(03)00057-5

Espada, M. C. y Grau, C. (2012). Estrategias de afrontamiento en padres de niños con cáncer. Psicooncología, 1(9), 25-40. http://dx.doi.org/10.5209/rev_PSIC.2012.v9.n1.39136

Frydenberg, E. (1997). Adolescent coping. Theoretical and research perspectives.Londres: Routledge.

García-Martín, M. A. y Jiménez-Hernández, M. (1995). El estrésinfantil. En M. Jiménez-Hernández (Ed.). Psicopatología Infantil. (2 ${ }^{\mathrm{a}}$ ed., pp. 85-98). Málaga: Aljibe D.L.

Giménez-Gualdo, A. M., Arnaiz-Sánchez, P. y Maquilon-Sánchez, J. J. (2013). Causas, medios y estrategias de afrontamiento de la agresión online en escolares de Murcia (España). Texto Livre: Linguagem e Tecnologia, 6(2), 1-17.

Hernández-Pereyra, Y. G. (2004). Percepción de riesgo volcánico y estrategias de afrontamiento de habitantes de zonas rurales y urbanas entorno al Popocatépetl. (Tesis de Licenciatura). Recuperado de: http://catarina.udlap.mx/u_dl_a/tales/documentos/lps/ hernandez_p_yg/ 
Lazarus, R. S. y Folkman, R. S. (1986). Stress appraisal and coping. New York, NY: Springer Publishing Company. (Traducción española: Barcelona, Martínez Roca, 1989).

Massone, A. y González, G. (2003).Estrategias de afrontamiento (coping) y su relación con el logro académico en matemática y lengua en adolescentes de noveno año de Educación General Básica. Revista Iberoamericana de Educación, 1-8. Recuperado de: http://www.rieoei.org/deloslectores/378Massone.PDF

Montero, I. y León, O. (2007).A guide for naming research studies in Psychology. International Journal of Clinical and Health Psychology, 7(3), 847-862. Recuperado de:http://www.redalyc.org/pdf/337/33770318.pdf

Morales, F. M. y Trianes, M. V. (2012). Afrontamiento en la infancia. Málaga: Aljibe.

Morales-Rodríguez, F. M., Trianes, M. V., Blanca, M. J., Miranda, J., Escobar, M. y Fernández-Baena, F. J. (2012). Escala de afrontamiento para niños (EAN): propiedades psicométricas. Anales de psicología, $2(28)$ 475-483. http://dx.doi.org/10.6018/analesps.28.2.136221

Rostosky, S., y Riggle, E. (2015). It takes a village, people! Happy together: Thriving as a same-sex couple in your family, workplace, and community. (pp. 113-118) American Psychological Association, Washington, DC. Retrieved from http://0-search.proquest.com.jabega.uma.es/docview/1 661993230 ?accountid $=14568$

Rovella, A. y Sans, M. (2003). Predictores de éxito académico en estudiantes universitarios. Revista Electrónica de Psicología Política, 6, 1-1. Recuperado de: http://www.psicopol.unsl.edu.ar/junio04_nota10.htm

Sánchez, J., Segovia, I. y Miñán, A. (2011). Exploración de la ansiedad hacia las matemáticas en los futuros maestros de Educación Primaria. Revista de currículum y formación del Profesorado, 15(3), 297-312. Recuperado de: http://www.ugr.es/ recfpro/rev153COL6.pdf

Seiffge-Krenke, I. (2000). Causal links between stressful events, coping style, and adolescent symptomatology. Journal of Adolescence, 23, 675-691. http://dx.doi.org/10.1006/jado.2000.0352

Seiffge-Krenke, I., y Shulman, S. (1990). Coping style in adolescence.Journal of Cross-cultural Psychology, 2, 351-337.http://dx.doi.org/10.1177/002202219021300 6

Trianes, M. V. (1999). Estrés en la Infancia. Madrid: Narcea ediciones. 